\title{
Intervertebral disc degeneration induced by long-segment in-situ immobilization: a macro, micro, and nanoscale analysis
}

\author{
Yan-Jun Che ${ }^{1,2}$, Hai-Tao Li', Ting Liang ${ }^{1}$, Xi Chen ${ }^{1}$, Jiang-Bo Guo ${ }^{1}$, Hua-Ye Jiang ${ }^{1}$, Zong-Ping Luo ${ }^{1 *}$ (D) \\ and Hui-Lin Yang ${ }^{1}$
}

\begin{abstract}
Background: Cervical spine fixation or immobilization has become a routine treatment for spinal fracture, dislocation, subluxation injuries, or spondylosis. The effects of immobilization of intervertebral discs of the cervical spine is unclear. The goal of this study was to evaluate the effects of long-segment in-situ immobilization of intervertebral discs of the caudal vertebra, thereby simulating human cervical spine immobilization.

Methods: Thirty-five fully grown, male Sprague-Dawley rats were used. Rats were randomly assigned to one of five groups: Group A, which served as controls, and Groups B, C, D, and E, in which the caudal vertebrae were in-situ immobilized using a custom-made external device that fixed four caudal vertebrae (Co7-Co10). After 2 weeks, 4 weeks, 6 weeks, and 8 weeks of in-situ immobilization, the caudal vertebrae were harvested, and the disc height, the T2 signal intensity of the discs, disc morphology, the gene expression of discs, and the structure and the elastic modulus of discs was measured.

Results: The intervertebral disc height progressively decreased, starting at the 6 th week. At week 6 and week 8, disc degeneration was classified as grade III, according to the modified Pfirrmann grading system criteria. Long-segment immobilization altered the gene expression of discs. The nucleus pulposus showed a typical cell cluster phenomenon over time. The annulus fibrosus inner layer began to appear disordered with fissure formation. The elastic modulus of collagen fibrils within the nucleus pulposus was significantly decreased in rats in group $\mathrm{E}$ compared to rats in group $\mathrm{A}(p<0.05)$. On the contrary, the elastic modulus within the annulus was significantly increased in rats in group $\mathrm{E}$ compared to rats in group $\mathrm{A}(p<0.05)$.

Conclusion: Long-segment in-situ immobilization caused target disc degeneration, and positively correlated with fixation time. The degeneration was not only associated with changes at the macroscale and microscale, but also indicated changes in collagen fibrils at the nanoscale. Long-segment immobilization of the spine (cervical spine) does not seem to be an innocuous strategy for the treatment of spine-related diseases and may be a predisposing factor in the development of the symptomatic spine.
\end{abstract}

Keywords: Intervertebral disc degeneration, Immobilization, Cervical spine, Fixation, Biomechanics, Rat model

\footnotetext{
* Correspondence: zongping_luo@yahoo.com

${ }^{1}$ Orthopaedic Institute, Department of Orthopaedics, The First Affiliated

Hospital of SooChow University, 708 Renmin Rd, Suzhou, Jiangsu 215006,

People's Republic of China

Full list of author information is available at the end of the article
}

(c) The Author(s). 2018 Open Access This article is distributed under the terms of the Creative Commons Attribution 4.0 International License (http://creativecommons.org/licenses/by/4.0/), which permits unrestricted use, distribution, and reproduction in any medium, provided you give appropriate credit to the original author(s) and the source, provide a link to the Creative Commons license, and indicate if changes were made. The Creative Commons Public Domain Dedication waiver (http://creativecommons.org/publicdomain/zero/1.0/) applies to the data made available in this article, unless otherwise stated. 


\section{Background}

Previous studies have shown that disc degeneration is closely associated with low back pain (LBP) [1, 2]. The mechanical environment of the intervertebral disc (IVD) at least in part determines the rate of disc degeneration. However, in terms of overload and "wear and tear" theory, the mechanical environment around the nucleus pulposus (NP) and annulus fibrosus (AF) is more harsher, so that damage to the disc cannot be completely recovered [3]. In previous studies, the degeneration model of articular cartilage induced by immobilization has been clearly demonstrated [4-9], however, the IVD degeneration induced by immobilization in human spine is still controversial. Due to fractures of the cervical spine, dislocations and cervical spondylosis, in order to restore the stability of the spine (cervical spine), a cervical extension collar, brace, or Halo-vest immobilization may be required. However, after release of immobilization, complications may remain, including stiffness of the neck and restricted movement $[10,11]$. It cannot be ruled out that the immobilization apparatus was a causal factor to the complications, and the underlying mechanism that is a factor in these complications has yet to be identified. In this study, three caudal vertebrae (including two discs) were fixed, this method manufactured reconstitution alterations that were similar to those found using static compression, but with less changes in configuration and synthesis [12]. Immobilization using fixation of two caudal vertebrae, including one disc, downregulated the expression of anabolic genes [13]. The hypothesis that movement increases the conveyance of nutrients and metabolites in the disc was recently investigated, and it was found that essential nutrients are transported through diffusion and convection [14]. Moreover, it was suggested that a "pumping" effect would accelerate the conveyance of molecules larger than that of sulfate ions. Several studies have shown the effect induced by short-segment immobilization (usually fixation is less than/or equal to three caudal vertebrae, including two discs). Because of the anatomical features of the cervical vertebrae (C4-7 intervertebral discs is an easier segment to degenerate or protrude), cervical spine immobilization or fixation (a brace or Halo-Vest fixator) is more inclined to overall fixation (similar to long tube fixation). However, the effect of long-segment in-situ immobilization and the underlying mechanism involved in the onset of complications remains unknown.

The purpose of this study is to identify the effect of long-segment in-situ immobilization (fixation of four caudal vertebrae, including three discs) caused by biochemical composition, gene expression, matrix reconstruction, and cellular responses, and to assess the effects of long-segment in-situ immobilization on intervertebral discs of the caudal vertebra, thereby simulating human cervical spine immobilization. Therefore, we hypothesize: 1) intervertebral disc degeneration is induced by long-segment in-situ immobilization; 2) intervertebral disc degeneration positively correlates with immobilization time; 3) the mechanism involved in complications of cervical spine long-segment in-situ immobilization can be explained at least in part by intervertebral disc degeneration.

\section{Methods}

In this study, thirty-five fully grown, 3-month-old male Sprague-Dawley rats were used [15]. Animals were randomly assigned to one of five groups (Table 1). Group A ( $n=7$ rats, the caudal vertebrae were instrumented with K-wires only, Fig. 1a) which served as controls. In the other four groups, vertebrae were immobilized using a custom-made external device to fix four caudal vertebrae (Co7-Co10). After 2 weeks ( $n=7$ rats, Group B), 4 weeks ( $n=7$ rats, Group C), 6 weeks ( $n=7$ rats, Group D), and 8 weeks ( $n=7$ rats, Group E) of immobilization, animals were euthanized and the caudal vertebrae were harvested for further analysis. The disc space was measured using radiography [16], and MRI qualitative analysis according to the modified Pffirmann scale [17]. Next, an experienced radiologist and a senior director experienced in spines analyzed the MRI-derived data (Table 2). Histological evaluation was based on the grading system developed by Han et al. [18]. Disc anabolic (collagen I, collagen II, aggrecan) and expression of catabolic genes (MMP3, MMP13, ADAMTs-4) were measured, respectively. Four K-wires were fixed in parallel using two aluminum alloy cuboids, which do not compress or stretch the experimental disc (Fig. 1b).

\section{Histological analysis}

After immobilization for 2, 4, 6, and 8 weeks, respectively, the rats were examined by X-ray and MRI analysis. Then, the animals were euthanized by an excess of isoflurane (isoflurane, RWD Life science co. Shenzhen, China). The target discs Co7-Co8, Co8-Co9, Co9-Co10 were harvested, fixed in $10 \%$ buffered formalin solution (Shanghai Yuanye Bio-Technology Co. Ltd., Shanghai, China) for $24 \mathrm{~h}$, and decalcified in $10 \%$ ethylenediaminetetraacetic acid (EDTA) (Biosharp, Hefei, China) for 30 days. The discs were then paraffin-embedded (Leica,

Table 1 Summary of study design

\begin{tabular}{lll}
\hline Group & Instrumented level (Co7-Co10) & No. of Animals \\
\hline A (Control) & Instrumented with K-wires only & 7 \\
B & Imm-2 week & 7 \\
C & Imm-4 week & 7 \\
D & Imm-6 week & 7 \\
E & Imm-8 week & 7
\end{tabular}

$\mathrm{Imm}$ indicates immobilization, Co indicates Coccygeal spine 


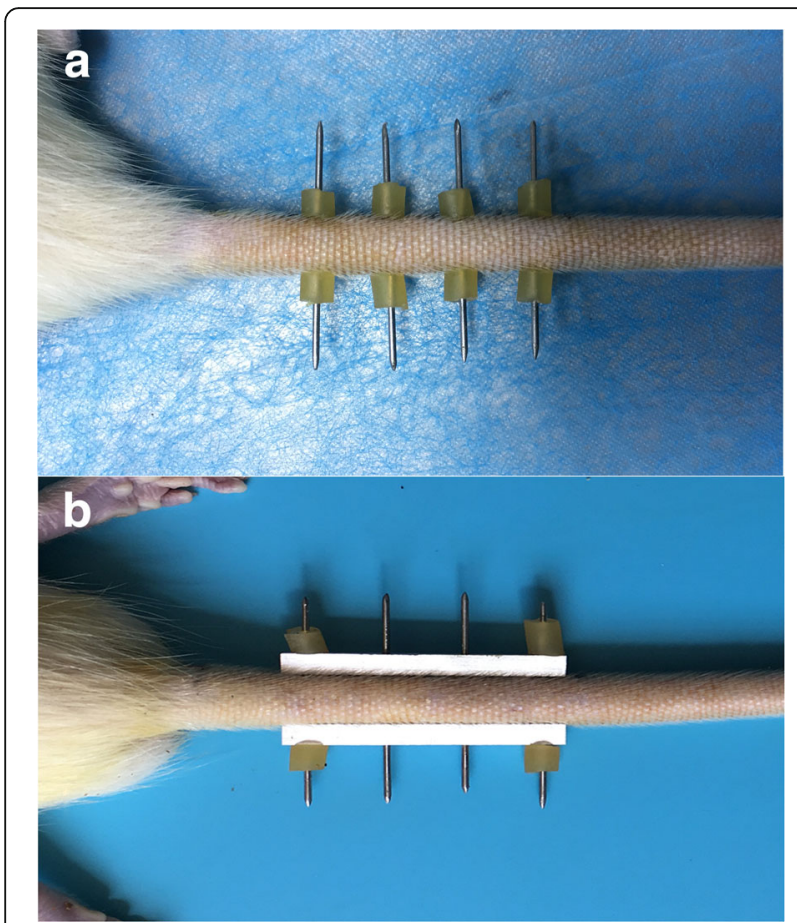

Fig. 1 Animal model. a. the caudal vertebrae were instrumented with K-wires only, which served as controls. $\mathbf{b}$. the caudal vertebrae were immobilized using a custom-made external device to fix four caudal vertebrae (Co7-Co10). Four K-wires (50 mm in length and $1.2 \mathrm{~mm}$ in diameter) were fixed in parallel using two aluminum alloy cuboids (43 mm in length, $4 \mathrm{~mm}$ in width, net weight $5.0 \mathrm{~g}$, the hole spacing is $12 \mathrm{~mm}$ ), which do not compress or stretch the experimental discs

Richmond, USA), and sectioned using a histotome (Leica, Heidelberger, Germany). For histological analysis, sections $(5 \mu \mathrm{m})$ were stained with hematoxylin/eosin (Beijing BiotoppedScience \& Technology Co. Ltd., Beijing, China), whereas for AFM scanning, 10-20 $\mu \mathrm{m}$ sections were used. Histological images were visualized using a binocular microscope (XSP-2CA, Shanghai, China), and changes in the AF were assessed using a grading scale of the stained images at a magnification of $200 \times$ as described by Masuda et al. [16]. The number of cells in the NP was scored by counting from the hematoxylin/eosin stained images at a magnification of $400 \times$.

Table 2 Modified Magnetic Resonance Imaging Pfirrmann

\begin{tabular}{llll}
\multicolumn{2}{l}{ Grading } & \\
\hline Grade & Structural Changes Within NP & Signal Intensity & IVDH \\
\hline I & Homogenous and bright & Hyperintense & Normal \\
II & Heterogeneous & Intermediate & Normal \\
III & Heterogeneous and gray & Intermediate & Decreased \\
IV & Heterogeneous and black & Hypointense & Decreased or \\
& & & collapsed \\
\hline
\end{tabular}

NP indicates nucleus pulposus, IVDH indicates Intervertebral Disc Height

\section{Gene expression analysis by RT-PCR}

For each specimen, (35 rats, 1 disc levels, AF and NP; $n=70)$, total RNA was extracted using TRIzol $^{\oplus}$ reagent and a total of $1 \mu \mathrm{g}$ of total RNA was reverse-transcribed using the Revert Aid First Strand cDNA Synthesis Kit (Thermo Fisher Scientific; Waltham, MA, USA). To quantify mRNA expression, an amount of cDNA that was equivalent to $50 \mathrm{ng}$ of total RNA was amplified by realtime PCR using the iTaqTM Universal SYBR ${ }^{\odot}$ Green Supermix kit (Bio-Rad, Hercules, CA, USA) [19]. Transcript levels of anabolic genes (collagen I, collagen II, aggrecan) and catabolic genes (MMP3, MMP13, ADAMTs-4) were evaluated. GAPDH served as an internal standard. Primer sequences are presented in Table 3. Rt-PCR was performed on a CFX96TM rt-PCR System (Bio-Rad, Hercules, CA, USA) following the manufacturer's guidelines. Relative transcript levels were calculated as $\mathrm{X}=2-\Delta \Delta \mathrm{Ct}$, in which $\Delta \Delta \mathrm{Ct}=\Delta \mathrm{E}-\Delta \mathrm{C}$, $\Delta \mathrm{E}=\mathrm{Ctexp}-\mathrm{CtGAPDH}$, and $\Delta \mathrm{C}=\mathrm{Ctct} 1-\mathrm{CtGAPDH}$ [19].

\section{AFM imaging and nano-mechanical testing}

AFM scanner (Dimension ICON, Bruker, USA) was used at atmospheric pressure [20]. The structure and the elastic modulus of individual collagen fibrils within intervertebral discs Co9-Co10 were tested at the nanoscale using AFM in week 2, 4, 6, and 8, respectively. A total of

Table 3 Primers and Probes for Real-Time RT-PCR

\begin{tabular}{|c|c|}
\hline Target Gene & Sequence $\left(5^{\prime} \rightarrow 3^{\prime}\right)$ \\
\hline \multicolumn{2}{|l|}{ GAPDH } \\
\hline Forward: & AGA CAG CCG CAT CTT CTT GT \\
\hline Reverse: & TAC TCA GCA CCA GCA TCA CC \\
\hline \multicolumn{2}{|l|}{ Collagen I } \\
\hline Forward: & ATG TTC AGC TाT GTG GAC \\
\hline Reverse: & GGA TGC CAT CTT GTC CAG \\
\hline \multicolumn{2}{|l|}{ Collagen \|I } \\
\hline Forward: & CCT GGA CCC CGT GGC AGA GA \\
\hline Reverse: & CAG CCA TCT GGG CTG CAA AG \\
\hline \multicolumn{2}{|l|}{ Aggrecan } \\
\hline Forward: & AGG ATG GCT TCC ACC AGT GC \\
\hline Reverse: & TGC GTA AAA GAC CTC ACC CTC C \\
\hline \multicolumn{2}{|l|}{ MMP-3 } \\
\hline Forward: & TCT TCC TCT GAA ACT TGG CG \\
\hline Reverse: & AGT GCT TCT GAA TGT CCT TCG \\
\hline \multicolumn{2}{|l|}{ MMP-13 } \\
\hline Forward: & GCA GCT CCA AAG GCT ACA A \\
\hline Reverse: & CAT CAT CTG GGA GCA TGA AA \\
\hline \multicolumn{2}{|l|}{ ADAMTS-4 } \\
\hline Forward: & CTT CGC TGA GTA GAT TCG TGG \\
\hline Reverse: & AGT TGA CAG GGT TTC GGA TG \\
\hline
\end{tabular}


thirty-five collagen fibrils from each rat were tested. Both AFM imaging and nano-mechanical testing were conducted at a scanning rate of $1 \mathrm{~Hz}$ using a Scan Asyst-Air probe, a curvature radius of $5 \mathrm{~nm}$, and a force constant of $0.4 \mathrm{~N} / \mathrm{m}$.

\section{Statistical analysis}

Experimental data are presented as the mean \pm standard deviation (SD). Significant differences between study groups were obtained by using a one-way analysis of variance (ANOVA) with Fisher's Partial Least-Squares Difference (PLSD) to analyze the influence of immobilization loading and time. Statistical significance was set at $p \leq 0.05$.

\section{Results}

All 35 rats successful completed the 8 week study. At the beginning of the study, the average body weight was $400 \mathrm{~g}$. Body weight increased to $405 \mathrm{~g}$ after 28 days, and was $411 \mathrm{~g}$ at the end of the study. This indicated that rats gained weight over time and that the surgery did not affect normal growth and development. The device that was used for immobilization weighted roughly $5.0 \mathrm{~g}$ and was well tolerated by the rats, as evidenced by their ability to lift and easily move their tails with the devices attached. Although the segments within the apparatus were largely immobilized, rats were able to move and control their tails both proximal and distal to the devices.

\section{Histology and morphology}

The intervertebral disc height progressively decreased with time. A significant decrease was observed in rats in group D and group $\mathrm{E}$ with time as shown in Fig. 2A. The percentage of intervertebral disc space height in groups A was significantly higher compared to that in group D and E (Fig. 2B) $(p<0.05)$. The disc thickness was significantly different between rats in group A compared to rats in group $\mathrm{D}(p=0.021)$, group $\mathrm{A}$ vs group $\mathrm{E}$ $(p<0.0001)$, and group $\mathrm{B}$ vs group $\mathrm{E}(p=0.028)$. At 8 weeks after spine immobilization, modified Pfirrmann grades of I, II, III, and IV were found in 12, 9, 14, and 0 rats, respectively. At week 6 and 8, the intervertebral disc degeneration was classified as grade III, based on the modified Pfirrmann grading system criteria. A total of 14 out of 35 discs had deteriorated between day 0 and 8 weeks after immobilization. A modified Pfirrmann grade of IV was not found in any of the animals examined.

Histological assessments were performed based on the grading system [18]. Previous studies have shown degeneration changes in immobilization IVD [12, 13, 21]. The histological changes found in this study were in line with the changes described by other groups [13, 21]. However, no significant decrease was found in the number of cells in the nucleus pulposus among rats in group A, B,
$\mathrm{C}, \mathrm{D}$, and $\mathrm{E}(p=0.370)$. In contrast, the number of cells in the nucleus pulposus was slightly increased over time in rats in group $\mathrm{D}$ and $\mathrm{E}(p>0.05)$. Moreover, the cluster formation of the nucleus pulposus cells became obvious. Cells within these clusters maintained their typical morphology with a polymorphonuclear shape, intracellular vacuoles, and stellar nuclei. Moreover, the Extracellular Matrix (ECM) progressively increased and the granules were shriveled. The inner layer of AF appear to be progressive disorders and hyperplasia (Fig. 3f-j). Round or ovoid-shaped chondrocytes infiltrated the AF, leading to the formation of cartilage-resembling tissue bordering the NP. This cartilage-resembling tissue could clearly be distinguished from the NP, displaying a different cell morphology and a matrix that was more intensely stained by hematoxylin/eosin (Fig. 3a-j). Characteristic AFM images of collagen fibrils from IVD of NP and AF for every group are presented in Fig. 4A. Within the NP, the elastic modulus of collagen fibrils progressively decreased (Fig. 4Ba), and was significantly different in rats in group A compared to rats in group $\mathrm{E}(p<0.001)$, group $\mathrm{A}$ vs group $\mathrm{D}$ $(p<0.05)$, group $\mathrm{B}$ vs group $\mathrm{E}(p<0.05)$, and group $\mathrm{C}$ vs group $\mathrm{E}(p<0.05)$. Moreover, a gradual increase was found within the AF (Fig. 4Bb), and was significantly different between the elastic modulus of collagen fibrils of rats in group A compared to rats in group $\mathrm{E}(p<0.001)$, group $\mathrm{B}$ vs group $\mathrm{E}(p<0.001)$, and group $\mathrm{C}$ vs group $\mathrm{E}(p<0.05)$.

\section{Gene expression}

In-situ immobilization affected the anabolic and catabolic gene expression of discs. The disc gene expression of Group A was consistent with those of the internal control discs. The trend of gene expression including downregulation of collagen II and aggrecan $(p<0.05$ for both), upregulation of collagen I, MMP3, MMP13, ADAMTs-4 of nucleus pulposus in Group B, C, D, and E, with the exception of group A ( $p<0.05$ all) (Fig. 5A(A-F)). Moreover, downregulation of collagen I $(p<0.05)$, upregulation of collagen II of AF in Group B, C, D, and E, and with the exception of group $\mathrm{A}$ (all $p<0.05)$, but did not show a significant effect on gene expression levels of aggrecan, MMP3, MMP13, and ADAMTs-4 of AF in Group A, B, C, D, and E $(p>0.05)$ (Fig. 5B(A-F)).

\section{Discussion}

Abnormal mechanically conditions are crucial contributing factors in IVD degeneration, however genetic factors may also play a significant role $[22,23]$. This study is the first to describe the changes in intervertebral discs in different periods of time during in-situ immobilization of caudal vertebrae in rats, based on a macro, micro, and nanoscale change analysis.

The complications of spine immobilization that are associated with halo-vest or a brace treatment have been 

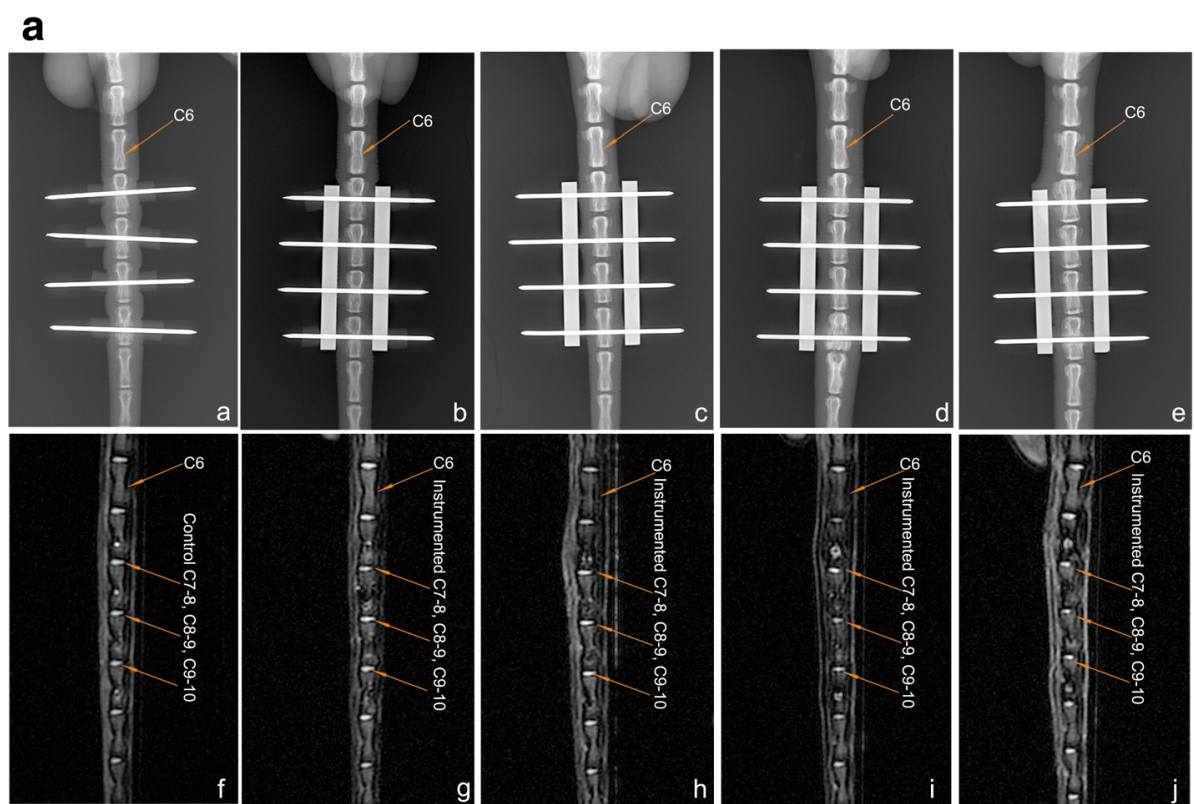

control

Imm 2 weeks

Imm 4 weeks

Group A Group B Group C

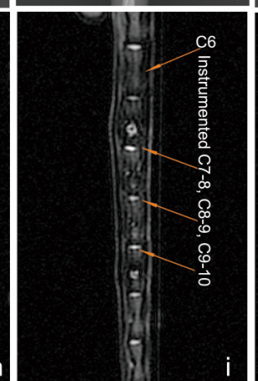

Imm 6 weeks Group D

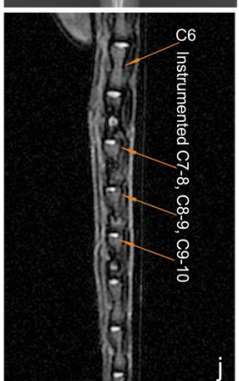

Imm 8 weeks Group E

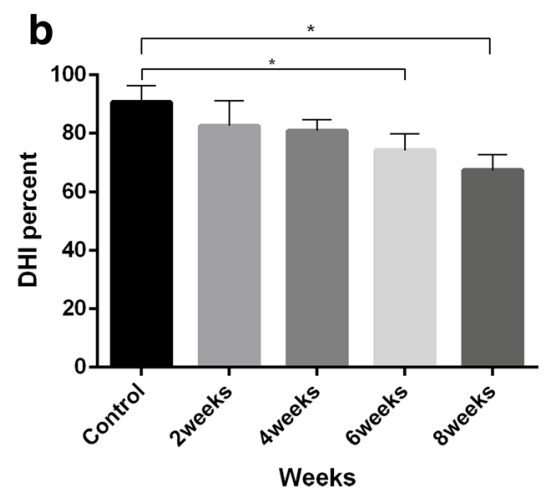

Fig. 2 A. The disc space and T2 signal intensity were measured using radiography and MRI Scans. Radiographs: (a e) were obtained under anesthesia using a digital, self-contained cabinet x-ray machine (exposure time: 10 s, 26 kV). a (Group A) which served as controls, b e (Group B E) as shown in the figure, over time, progressive loss of disc height, $d$ and e more obvious. MRI Scans: (f j) (Scanning Sequence: FRFSE-XL, Slice thickness:1.4 mm, Interlayer Spacing: $5 \mathrm{~mm}$ ) uses magnetic waves to create pictures to determine nucleus pulposus size and hydration status according to T2 signal intensity. Over time, the T2 signal intensity progressive decrease, as described above, $\mathrm{i}$ and $\mathrm{j}$ more serious. The IVD degeneration was classified as grade III. Imm indicates immobilization. B. The intervertebral disc height assessment based on radiographs. In control group (group A), imm-2 weeks (group B), and imm-4 weeks (group C), the intervertebral disc space height was slightly decreased, postoperatively, and this reduction was significant starting at the 6 th week (group D). (*) indicates significant difference from other groups discs $(p<0.05)$

intensively investigated [24-27]. These studies showed that complications included osteomyelitis and heterotopic ossification etc. It has not yet been analyzed how halo immobilization leads to the complications associated with intervertebral disc degeneration. In our study, we have assessed the effects of long-segment in-situ immobilization on the intervertebral disc of the caudal vertebra, thereby simulating human cervical spine immobilization. Elliott et al. [28] demonstrated a link between intervertebral discs and body weight that was similar in rats and humans. The rat-tail model was chosen as a model [12, 29-31], in our study, the mechanical environment of the IVD that allows for precise control. Thereby we can rule out the effect of mechanical manipulation from other contributing factors.

A retrospective study in which the stress and activity of IVD degeneration was evaluated, demonstrated that mechanical intervention not only plays a role in changing the pressure or activity, however the application device also reduced the activity of the disc, which can result in IVD degeneration [21]. Our study showed that the the disc height and the MRI T2 signal strength of 


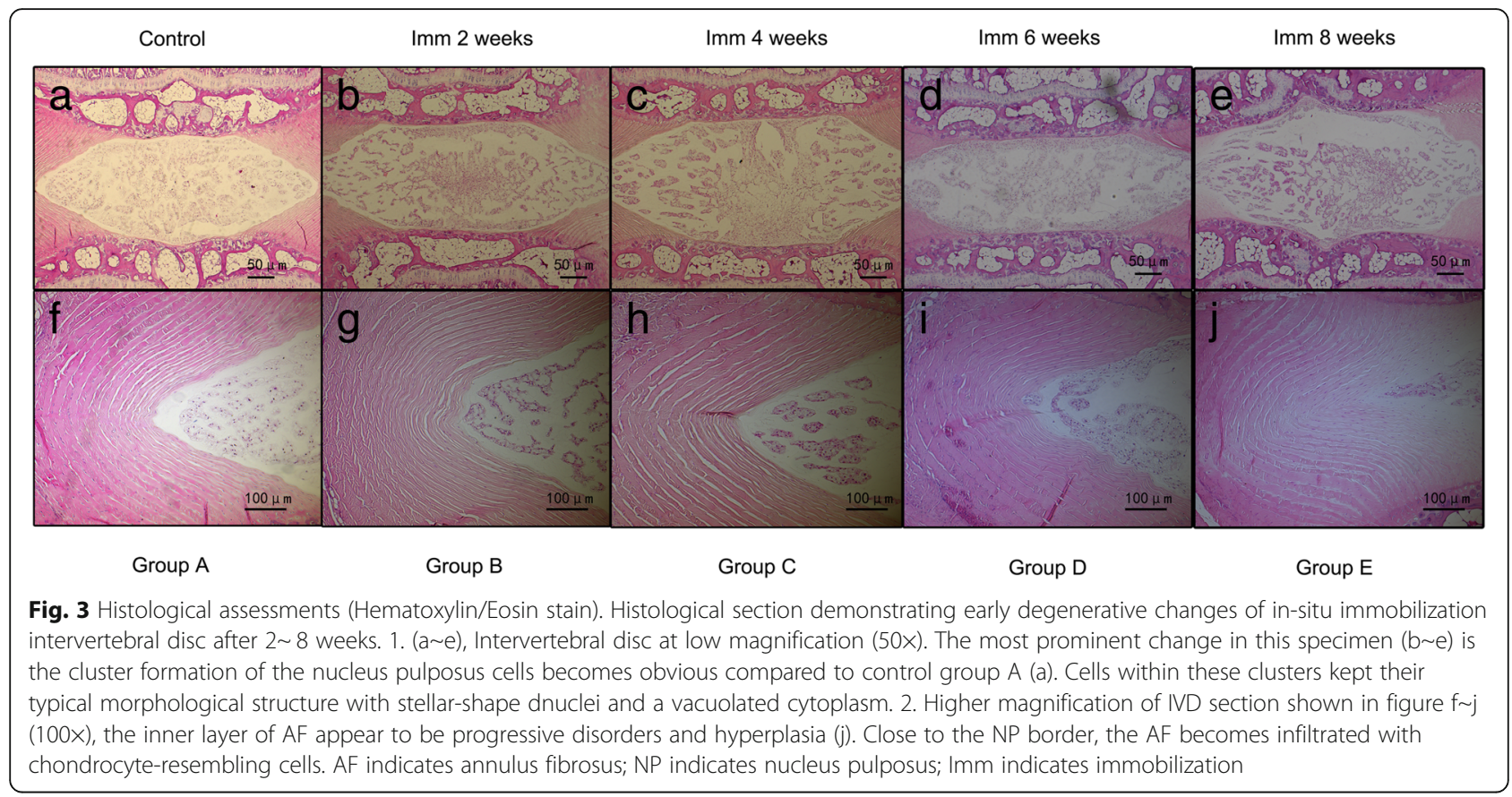

the experimental disc gradually decreased with fixation time.

Previous studies have shown that fixation triggered a degradation pattern in the IVD, such as downregulation of anabolic gene expression [13], and a decrease in glycosaminoglycan content [32]. Our findings further indicated that the effects of immobilization were more significant in the NP compared to the AF. The greatest effect was observed in group E, and combined with the loss of disc height, MRI T2 signal attenuation and IVD elastic modulus gradual decline over time, and confirmed that degeneration of IVD was not only present at the macroscale and microscale, but also at the nanoscale. These data suggested that immobilization can cause early stage degeneration of the IVD that progressed in severity over time. The long-term nature of this study that hypomobility has an effect on gene expression of the NP was more prominent compared to changes found in the AF. This discrepancy may be due to the different periods of immobilization time and abnormal mechanical conditions. In our study, we were the first to show direct evidence of metabolic response in the IVD in vivo associated with long-segment in-situ immobilization.

The AF with a highly organized structure, runs at angles of approximately $60^{\circ}$ to the spinal column [33]. Therefore, elastic fibers perform a very crucial role in the total mechanical properties of the AF [34]. In our previous study, we demonstrated the structure and biomechanics at the nanoscale level from different regions of the AF in loaded IVD [20]. This study revealed that the elastic modulus of nucleus pulposus collagen fibrils in group E was markedly decreased compared to group A (control group). Moreover, the elastic modulus was remarkably increased within the AF, and a significant difference was found in the elastic modulus of collagen fibrils over time. The results are consistent with the morphologic changes of NP and AF at the microscale as indicated by hematoxylin/eosin staining. Moreover, the tendency of gene expression of NP and AF were consistent with the former. Therefore, the results indicate that degradation was not only associated with the disorganization at the microscale, but also suggested modification of collagen fibrils at the nanoscale, which would directly change the mechanical environment around the cells of the AF and NP.

From what has been discussed above, in-situ immobilization creates a unique mechanical state that can cause disc degeneration. Previous studies have confirmed that too much or too little pressure/stretch contributes to a catabolism effect on intervertebral discs, in which hypomobility or excessive activities will increase the damage rate, leading to intervertebral disc on the load response of a U-shaped distribution [35]. It is generally accepted that immobilization osteoporosis (IOP) caused by partial fixation after fractures of the lower limb is a common complication in clinical therapeutics. Bed rest and immobilization time are independent predictors of low bone density in the hip [36]. According to Wolff's law, disuse osteoporosis is thought to be associated with a lack of mechanical forces $[37,38]$. We believe that hypomobility or immobilization caused degeneration of muscular ligaments and facet joints in surrounding spine tissue. However, we cannot exclude 


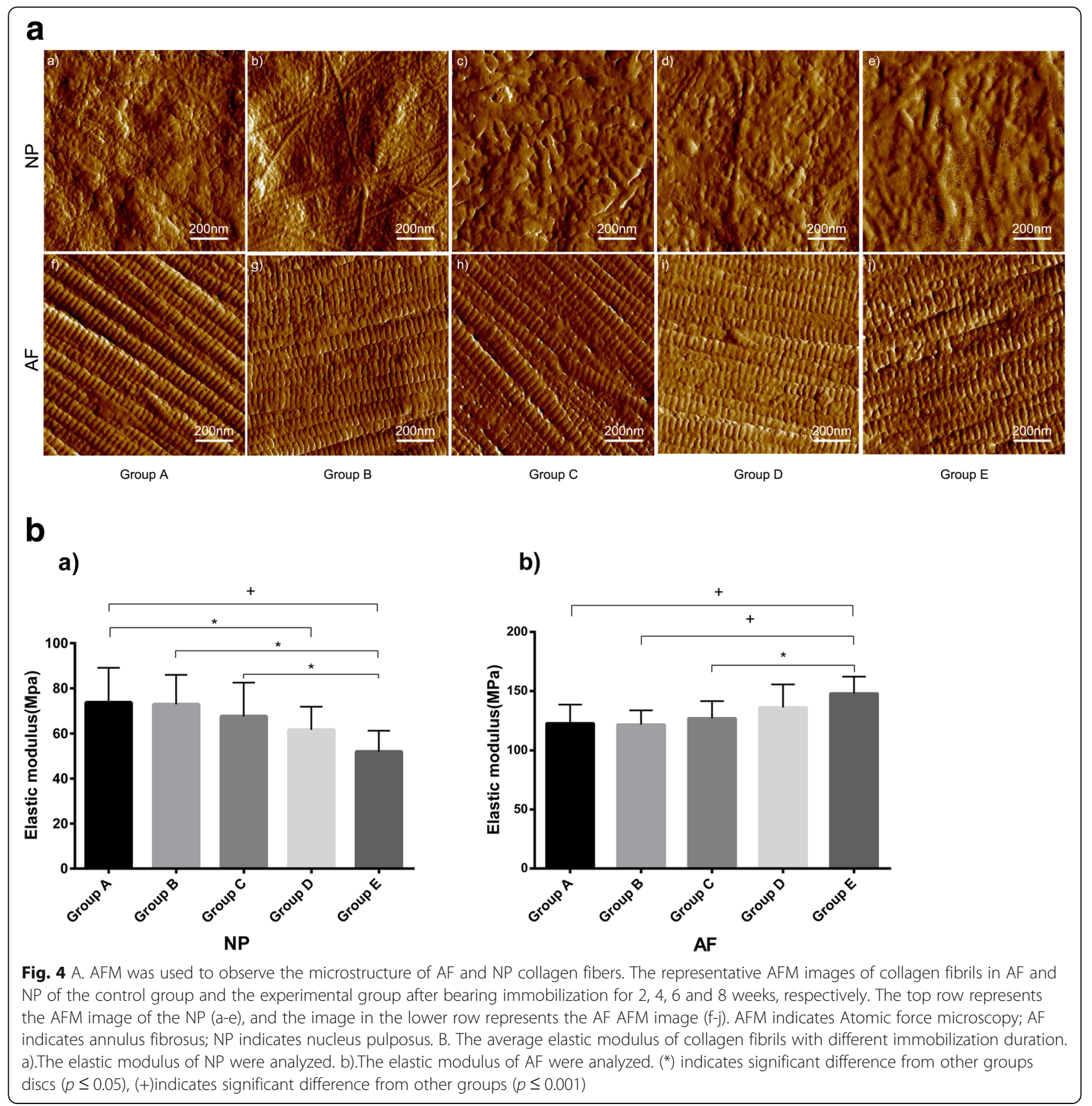

the possibility that the immobilization apparatus was a factor in the degeneration. Setton et al. showed that hypomobility produced a reduced stimulus to the metabolic activity of disc cells [39]. In an unloaded condition, the IVD swells and starts to lose proteoglycans [40]. Dynamic loading of a certain magnitude, frequency, and duration has been shown to maintain the ECM balance within the disc [41-44]. Static loading induces cell death and causes disc degradation [21, 45-47]. Choi et al. [48] insisted that spine fixation and endplate injury or fracture by internal transpedicular fixation without fusion plays a crucial role in IVD degradation. These studies suggested that hypomobility or immobilization and static loads are both not an innocuous mechanical environment to IVD, in contrast, an adverse effect was found. Ragab et al. [49] showed that cervical fusion resulted in increased strains at adjacent levels. However, long-segment cervical fusions (2 and 3-level fusions) compared to short-segment fusions (1-level fusions), increased the strain approximately 2 - to 3 -fold compared to the standard. These findings suggested that long-segment fusions or immobilization result in increased side effects compared to short-segment fusions or 


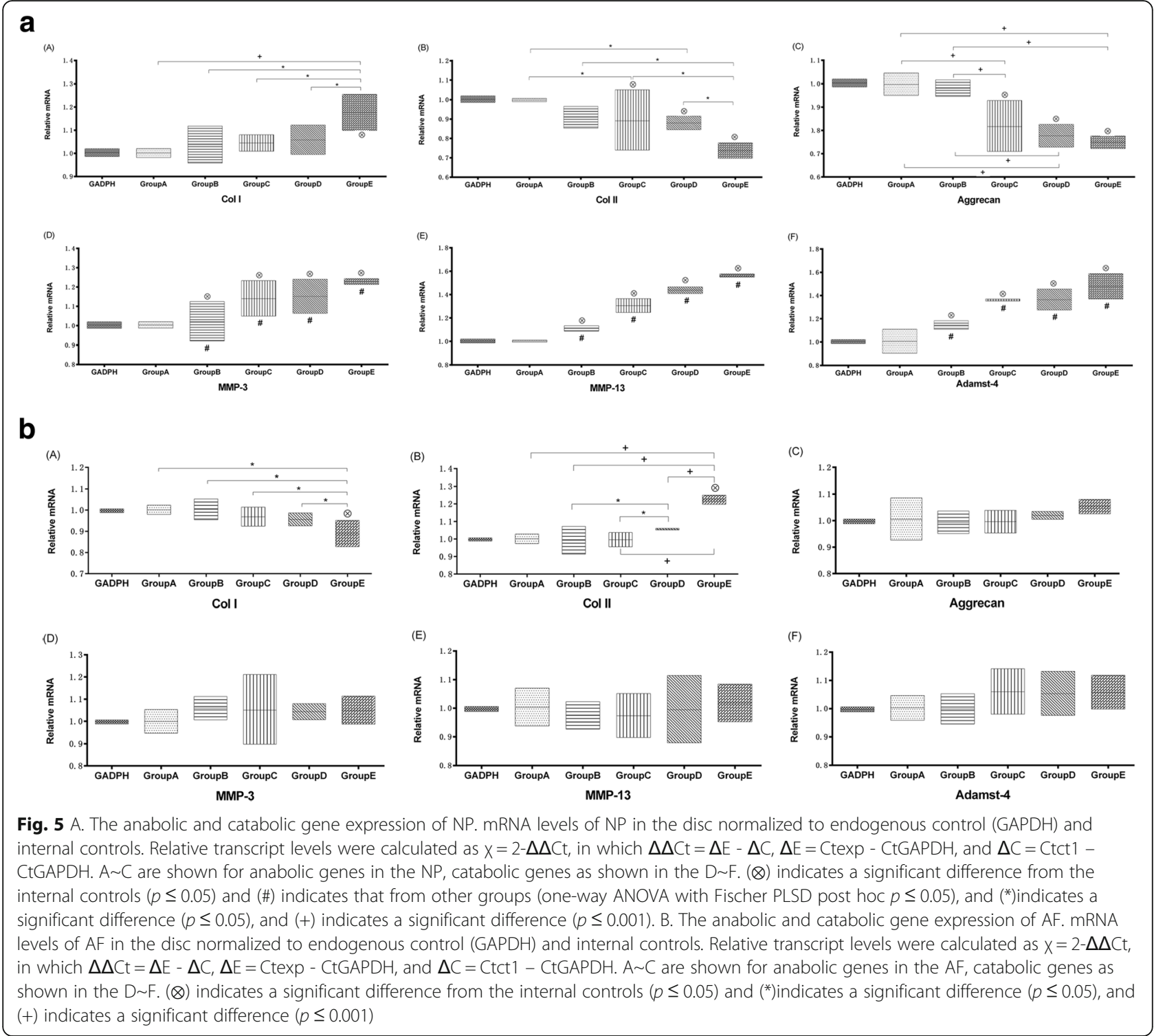

fixation. This indicates that the long-segment in-situ immobilization triggered a degradation pattern in the IVD. Therefore, in the clinic, it should be possible to reduce spine (cervical spine) long segmental immobilization, and spine (cervical spine) immobilization time ( $\leq 6$ weeks).

However, a limitation of this study was the difference between a rat tail disc and a human cervical disc in terms of biochemical composition, molecular composition, and biomechanical capabilities. We used the rat caudal vertebral model to simulate human cervical spine IVD changes. While not completely equal to human intervertebral disc changes, the rat model is the currently accepted model and was easier to build and replicate than alternatives. More importantly, it provided relevant experimental specimens and related parameters of IVD that human cervical spine cannot provide. It also provides us with detailed experimental data and theoretical basis for further elaborating the effect of immobilisation on intervertebral disc.

\section{Conclusions}

In this study, long-segment in-situ immobilization caused target disc degeneration, and positively correlated with fixation time. These findings showed that, over time, in-situ immobilization induced a progressive decrease in the intervertebral disc space height and T2 signal intensity of IVD. In addition, downregulation of collagen II, and upregulation of collagen I, aggrecanase, collagenase, stromelysin of $\mathrm{NP}$, and downregulation of collagen I, and upregulation of collagen II of AF were found. However, the effects on aggrecanase, collagenase, stromelysin of AF were not significant. These changes suggested that immobilization loading initiated a degenerative cascade, although this 
trend was significantly observed in the NP compared to AF. These results as well as the variation tendency of the elastic modulus of collagen fibrils within the NP and AF were confirmed. The discrepancy found with other studies, may be due to the different periods of immobilization and loading conditions. Histological analysis, including hematoxylin/eosin staining indicated that the NP and AF showed a progressive degeneration by in-situ immobilization. However, the comparison with overload conditions was moderate. This study evaluated how hypomobility of the spine may result in IVD degradation and spine lesions. The loading parameters chosen for our study were intended to imitate cervical spine long-segment in-situ immobilization in humans. The results indicated that the degeneration was not only associated with changes at the macroscale, microscale, but also indicated changes in collagen fibrils at the nanoscale, which would directly change the mechanical environment around the cells of the NP and AF. In conclusion, increasing our understanding of the pathogenesis and complications found after long-segment immobilization of the cervical spine, and how to optimize the use of external fixator devices are clearly warranted.

\section{Abbreviations}

AF: Annulus fibrosus; AFM: Atomic Force Microscope; Co: Coccygeal spine; ECM: Extracellular Matrix; EDTA: Ethylenediaminetetraacetic acid; IVD: Intervertebral disc; LBP: Low back pain; NP: Nucleus pulposus; PCR: Polymerase chain reaction

\section{Acknowledgements}

We thank Zong-Da Jin PhD, (Department of Public Health, ZheJiang medical College), and Yue Zhang PhD, (Department of Epidemiology, School of Public Health, Fudan University) for their help with the statistical analysis.

\section{Funding}

Funding for the study was provided by the National Natural Science Foundation of China (81320108018, 31570943, 31270995 and 81702146), Innovation and Entrepreneurship Program of Jiangsu Province, and the Priority Academic Program Development of Jiangsu Higher Education Institutions. The funding body did not influence the design of the study, the collection, analysis, or interpretation of the data, or the writing of the manuscript.

\section{Availability of data and materials}

The datasets used and/or analysed during the current study are available from the corresponding author on reasonable request.

\section{Authors' contributions}

YJC and ZPL initiated the study design. HTL, TL, XC, JBG and HYJ participated in the data collection and data analysis. YJC and ZPL participated in the data analysis and the preparation of the manuscript. HLY was an advisor. All authors have read and approved the final manuscript.

\section{Ethics approval and consent to participate}

All animal experiments were strictly performed under the guidelines of the Chinese Council for Animal Care, approved by the Animal Care Committee of the Laboratory Animal at School of Medicine, SooChow University(ECSU201700035).

\section{Consent for publication}

Not applicable.

\section{Competing interests}

The authors declare that they have no competing interests.

\section{Publisher's Note}

Springer Nature remains neutral with regard to jurisdictional claims in published maps and institutional affiliations.

\section{Author details}

${ }^{1}$ Orthopaedic Institute, Department of Orthopaedics, The First Affiliated Hospital of SooChow University, 708 Renmin Rd, Suzhou, Jiangsu 215006 People's Republic of China. ${ }^{2}$ Department of Orthopedics, Peace Hospital Affiliated to Changzhi Medical College, Changzhi, Shanxi, People's Republic of China.

Received: 21 November 2017 Accepted: 16 August 2018

Published online: 28 August 2018

\section{References}

1. Schwarzer AC, Aprill CN, Derby R, Fortin J, Kine G, Bogduk N. The relative contributions of the disc and zygapophyseal joint in chronic low back pain. Spine (Phila Pa 1976). 1994;19(7):801-6.

2. Buckwalter JA. Aging and degeneration of the human intervertebral disc. Spine (Phila Pa 1976). 1995;20(11):1307-14.

3. Urban JP, Roberts S. Development and degeneration of the intervertebral discs. Mol Med Today. 1995:1(7):329-35.

4. Hong SP, Henderson CN. Articular cartilage surface changes following immobilization of the rat knee joint. A semiquantitative scanning electronmicroscopic study. Acta Anat (Basel). 1996;157(1):27-40.

5. Leroux MA, Cheung HS, Bau JL, Wang JY, Howell DS, Setton LA. Altered mechanics and histomorphometry of canine tibial cartilage following joint immobilization. Osteoarthr Cartil. 2001;9(7):633-40.

6. Muller FJ, Setton LA, Manicourt DH, Mow VC, Howell DS, Pita JC. Centrifugal and biochemical comparison of proteoglycan aggregates from articular cartilage in experimental joint disuse and joint instability. J Orthop Res. 1994;12(4):498-508.

7. Narmoneva DA, Cheung HS, Wang JY, Howell DS, Setton LA. Altered swelling behavior of femoral cartilage following joint immobilization in a canine model. J Orthop Res. 2002;20(1):83-91.

8. Saamanen AM, Tammi M, Jurvelin J, Kiviranta I, Helminen HJ. Proteoglycan alterations following immobilization and remobilization in the articular cartilage of young canine knee (stifle) joint. J Orthop Res. 1990;8(6):863-73.

9. Setton LA, Mow VC, Muller FJ, Pita JC, Howell DS. Mechanical behavior and biochemical composition of canine knee cartilage following periods of joint disuse and disuse with remobilization. Osteoarthr Cartil. 1997;5(1):1-16.

10. Askins V, Eismont FJ. Efficacy of five cervical orthoses in restricting cervical motion. A comparison study. Spine (Phila Pa 1976). 1997;22(11):1193-8.

11. Clancy MJ. Clearing the cervical spine of adult victims of trauma. J Accid Emerg Med. 1999;16(3):208-14.

12. latridis JC, Mente PL, Stokes IA, Aronsson DD, Alini M. Compression-induced changes in intervertebral disc properties in a rat tail model. Spine (Phila Pa 1976). 1999;24(10):996-1002.

13. MacLean JJ, Lee CR, Grad S, Ito K, Alini M, latridis JC. Effects of immobilization and dynamic compression on intervertebral disc cell gene expression in vivo. Spine (Phila Pa 1976). 2003;28(10):973-81.

14. Urban JP, Holm S, Maroudas A, Nachemson A. Nutrition of the intervertebral disc: effect of fluid flow on solute transport. Clin Orthop Relat Res. 1982; (170):296-302. PMID: 7127960.

15. Hughes PC, Tanner JM. The assessment of skeletal maturity in the growing rat. J Anat. 1970;106(Pt 2):371-402.

16. Masuda K, Aota Y, Muehleman C, Imai Y, Okuma M, Thonar EJ, Andersson $\mathrm{GB}, \mathrm{An} \mathrm{HS}$. A novel rabbit model of mild, reproducible disc degeneration by an anulus needle puncture: correlation between the degree of disc injury and radiological and histological appearances of disc degeneration. Spine. 2005;30(1):5-14.

17. Pfirrmann CW, Metzdorf A, Zanetti M, Hodler J, Boos N. Magnetic resonance classification of lumbar intervertebral disc degeneration. Spine (Phila Pa 1976). 2001:26(17):1873-8.

18. Han B, Zhu K, Li FC, Xiao YX, Feng J, Shi ZL, Lin M, Wang J, Chen QX. A simple disc degeneration model induced by percutaneous needle puncture in the rat tail. Spine. 2008;33(18):1925-34. 
19. Liu X, Zhou L, Chen X, Liu T, Pan G, Cui W, Li M, Luo ZP, Pei M, Yang H, et al. Culturing on decellularized extracellular matrix enhances antioxidant properties of human umbilical cord-derived mesenchymal stem cells. Mater Sci Eng C Mater Biol Appl. 2016;61:437-48.

20. Liang T, Zhang LL, Xia W, Yang HL, Luo ZP. Individual collagen fibril thickening and stiffening of annulus Fibrosus in degenerative intervertebral disc. Spine (Phila Pa 1976). 2017;42(19):E1104-E1111. PMID: 28146016. https://doi.org/10.1097/BRS.0000000000002085.

21. Stokes IAF, latridis JC. Mechanical conditions that accelerate intervertebral disC degeneration: overload versus immobilization. Spine. 2004;29(23):2724-32.

22. Walter BA, Korecki CL, Purmessur D, Roughley PJ, Michalek AJ, latridis JC. Complex loading affects intervertebral disc mechanics and biology. Osteoarthr Cartil. 2011;19(8):1011-8.

23. Kroeber MW, Unglaub F, Wang H, Schmid C, Thomsen M, Nerlich A, Richter W. New in vivo animal model to create intervertebral disc degeneration and to investigate the effects of therapeutic strategies to stimulate disc regeneration. Spine (Phila Pa 1976). 2002;27(23):2684-90.

24. Baum JA, Hanley EN Jr, Pullekines J. Comparison of halo complications in adults and children. Spine (Phila Pa 1976). 1989;14(3):251-2

25. Kang M, Vives MJ, Vaccaro AR. The halo vest: principles of application and management of complications. J Spinal Cord Med. 2003;26(3):186-92.

26. Hossain M, McLean AN, Fraser MH. Outcome of halo immobilisation of 104 cases of cervical spine injury. Scot Med J. 2004;49(3):90-2.

27. Garfin SR, Botte MJ, Waters RL, Nickel VL. Complications in the use of the halo fixation device. J Bone Joint Surg Am. 1986;68a(3):320-5.

28. Elliott DM, Sarver JJ. Young investigator award winner: validation of the mouse and rat disc as mechanical models of the human lumbar disc. Spine. 2004;29(7):713-22.

29. Lotz JC, Colliou OK, Chin JR, Duncan NA, Liebenberg E. Compressioninduced degeneration of the intervertebral disc: an in vivo mouse model and finite-element study. Spine (Phila Pa 1976). 1998;23(23):2493-506.

30. Simunic DI, Broom ND, Robertson PA. Biomechanical factors influencing nuclear disruption of the intervertebral disc. Spine (Phila Pa 1976). 2001;26(11):1223-30.

31. Stokes IA, Aronsson DD, Spence H, latridis JC. Mechanical modulation of intervertebral disc thickness in growing rat tails. J Spinal Disord. 1998;11(3):261-5.

32. Ching $\mathrm{CT}$, Chow DH, Yao FY, Holmes AD. Changes in nuclear composition following cyclic compression of the intervertebral disc in an in vivo rat-tail model. Med Eng Phys. 2004;26(7):587-94.

33. Smith LJ, Fazzalari NL. The elastic fibre network of the human lumbar anulus fibrosus: architecture, mechanical function and potential role in the progression of intervertebral disc degeneration. Eur Spine J. 2009;18(4):439-48.

34. Fu LJ, Chen CS, Xie YZ, Yang JW, Sun XJ, Zhang P. Effect of a new annular incision on biomechanical properties of the intervertebral disc. Orthop Surg. 2016:8(1):68-74.

35. Lotz JC. Load and the spine. How does the goldilocks principle apply? Spine J. 2011;11(1):44-5.

36. Smith EM, Comiskey CM, Carroll AM. A study of bone mineral density in adults with disability. Arch Phys Med Rehabil. 2009;90(7):1127-35.

37. Adams MA, Dolan P. Biomechanics of vertebral compression fractures and clinical application. Arch Orthop Trauma Surg. 2011;131(12):1703-10.

38. Turner $\mathrm{CH}$. Three rules for bone adaptation to mechanical stimuli. Bone. 1998;23(5):399-407.

39. Setton LA, Chen J. Cell mechanics and mechanobiology in the intervertebral disc. Spine (Phila Pa 1976). 2004;29(23):2710-23.

40. Urban JP, Maroudas A. Swelling of the intervertebral disc in vitro. Connect Tissue Res. 1981;9(1):1-10.

41. Maclean JJ, Lee CR, Alini M, latridis JC. Anabolic and catabolic mRNA levels of the intervertebral disc vary with the magnitude and frequency of in vivo dynamic compression. J Orthop Res. 2004;22(6):1193-200.

42. MacLean JJ, Lee CR, Alini M, latridis JC. The effects of short-term load duration on anabolic and catabolic gene expression in the rat tail intervertebral disc. J Orthop Res. 2005;23(5):1120-7.

43. Walsh AJ, Lotz JC. Biological response of the intervertebral disc to dynamic loading. J Biomech. 2004;37(3):329-37.

44. Wuertz K, Godburn K, MacLean JJ, Barbir A, Donnelly JS, Roughley PJ, Alini $M$, latridis JC. In vivo remodeling of intervertebral discs in response to shortand long-term dynamic compression. J Orthop Res. 2009;27(9):1235-42.

45. Ariga K, Yonenobu K, Nakase T, Hosono N, Okuda S, Meng W, Tamura Y, Yoshikawa H. Mechanical stress-induced apoptosis of endplate chondrocytes in organ-cultured mouse intervertebral discs: an ex vivo study. Spine (Phila Pa 1976). 2003;28(14):1528-33.
46. Lotz JC, Chin JR. Intervertebral disc cell death is dependent on the magnitude and duration of spinal loading. Spine. 2000;25(12):1477-82

47. Lotz JC, Colliou OK, Chin JR, Duncan NA, Liebenberg E. 1998 Volvo award winner in biomechanical studies - compression-induced degeneration of the intervertebral disc: an in vivo mouse model and finite-element study. Spine. 1998;23(23):2493-506.

48. Choi W, Song S, Chae S, Ko S. Comparison of the extent of degeneration among the normal disc, immobilized disc, and immobilized disc with an endplate fracture. Clin Orthop Surg. 2017;9(2):193-9.

49. Ragab AA, Escarcega AJ, Zdeblick TA. A quantitative analysis of strain at adjacent segments after segmental immobilization of the cervical spine. J Spinal Disord Tech. 2006;19(6):407-10.
Ready to submit your research? Choose BMC and benefit from:

- fast, convenient online submission

- thorough peer review by experienced researchers in your field

- rapid publication on acceptance

- support for research data, including large and complex data types

- gold Open Access which fosters wider collaboration and increased citations

- maximum visibility for your research: over $100 \mathrm{M}$ website views per year

At BMC, research is always in progress.

Learn more biomedcentral.com/submissions 